\title{
A Case-Control Study of Relevance Between NOS2 rs2297518 Polymorphism with Preeclampsia Risk in the Chinese Han women
}

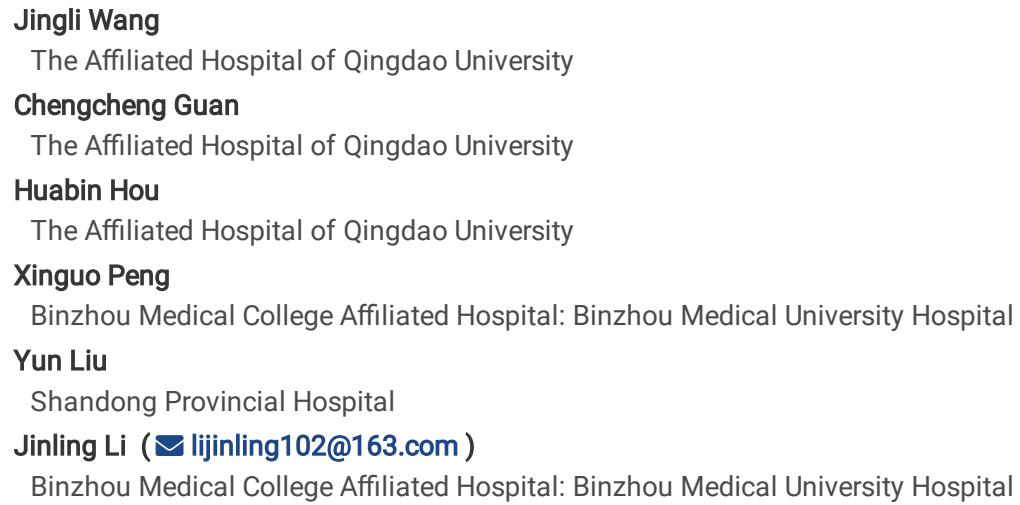




\section{Abstract}

Backgroud: Inducible Nitric Oxide Synthase (iNOS) acts on the contraction and expansion of blood vessels by mediating the synthesis of Nitric Oxide (NO), which is implicated in the pathophysiology of preeclampsia (PE) associated with systemic vasoconstriction. The polymorphism of NOS2, the gene of coding iNOS, can affect the function of protein. Therefore, we aimed to explore whether the polymorphism site rs2297518 in NOS2 is associated with susceptibility of $P E$ in a Chinese Han population.

Methods and Results:Genotyping the NOS2 rs2297518 polymorphism through TaqMan real-time fluorescence quantitative polymerase chain reaction (PCR) after DNA extraction from blood samples in this case-control study including 979 PE patients and 1187 healthy pregnant women. Using independent sample ttest and chi-square test to analyse clinical data and experimental results. We performed the odds ratios (ORs) and $95 \%$ confidence intervals (Cls) to estimate the degree of the association.There was no statistical significance in the genetic distributions for the polymorphism of rs 2297518 between the PE and control groups $\left(c^{2}=1.43, p=0.49\right.$ by genotype frequency, $c^{2}=0.85, p=0.36$, odds ratio $=1.07,95 \%$ confidence interval $0.92-1.25$ by allele frequency), and no differences among early/late-onset or mild/severe PE and controls was seen.

Conclusion: Our results indicated that the NOS2 rs2297518 polymorphism may be not play a major role in the susceptibility to PE in the Chinese Han population. Therefore, it is essential to test other polymorphisms of this gene to validate a potential relationship for susceptibility to PE.

\section{Introduction}

As a major cause of fetal growth restriction, preterm delivery and maternal death, preeclampsia (PE) is characterized by de novo development of hypertension and accompanied by proteinuria, which may progress into a multisystem disorder after 20 weeks gestation[1]. PE affects approximately $2 \sim 8 \%$ of all pregnant women in worldwide according to the World Health Organization. Although many therapeutic interventions have been proposed, the mortality and morbidity rates of PE patients remain as a challenge due to its unclear etiopathogenesis. The typical pathological manifestation of PE is systemic vasoconstriction, which may be related to abnormal changes in renal hemodynamics and NO level [2].

In normal physiological conditions, pregnant women have an obvious increase in renal plasma flow and glomerular filtration rate compared with ordinary women [3], which leads to the increased production of NO and expression of inducible NOS (iNOS) [4]. iNOS is a nitric oxide synthase, encoded by NOS2 and activated in inflammatory conditions by cytokines[5]. Besides, the expression of iNOS have been observed in villous stroma cells, which may play a protective role for the fetus to avoid infection [6]. However, other investigators have indicated that iNOS is involved in the occurrence of PE[7]. An animal test showed that increasing the iNOS expression of pregnant rats with placental ischemia led to vascular oxidative stress, and hypertension [7]. Besides, elevated vascular iNOS expression has been observed in the reduced uterine perfusion pressure(RUPP) rat model, a model that can present PE-like clinical symptoms [7]. When injected with an inhibitor of iNOS in this rat, PE features and increased level of iNOS was weaken [7]. Previous studies have indicated that the pathogenesis of placental ischemia in PE contributes to oxidative stress. In turn, reactive oxygen species (ROS) can react with NO produced by iNOS to form peroxynitrite in the development of PE $[8,9]$. Moreover, circulating pro-inflammatory cytokines (IL-1, IL-6, chemokine) can promote the increase of iNOS in PE patients compared to normal pregnancies[10]. This conclusion was also confirmed by another study that human umbilical vein endothelial cells (huvec) incubating with PE patient serum expressed higher iNOS mRNA compared with huvec stimulated by healthy pregnant women serum[11]. Analyzing the placenta iNOS expression of $32 \mathrm{PE}$ patients and 32 healthy pregnant women, researchers found the iNOS placenta expression had an obvious increase in the PE case group [12]. However, Faxén et al. presented a different result that the iNOS placenta expression manifested a decrease trend in PE patients [13].

$\mathrm{PE}$ is a disease of multiple factors and aberrent genes leading to this pregnancy complication. In recent years, researchers have begun to focus on the associations between candidate genes with PE susceptibility. A functional polymorphic site of rs2297518, located in exon 6 of NOS2[14], causes a base substitution from $\mathrm{G}$ to $\mathrm{A}$ at 2087 locos of its mRNA and leads to an amino acid change from serine to leucine (p.S696L), which can increase NOS2 activity by altering NOS2 protein function and confer higher NO production based on the A-allele[15]. Amaral et al. indicated a PE group had a higher A allele frequency of rs2297518 among the Brazilian population [16]. However, Seppo et al. stated this variant is not related to the susceptibility of hypertension in the Finnish population[14]. Therefore, this study aimed to explore whether the rs2297518 polymorphism has statistical different between a PE group and a control group of Chinese Han women and further find out the association between this polymorphism and susceptibility to PE.

\section{Materials And Methods}

\section{Study participants:}

We enrolled 979 PE patients and 1187 normal pregnant women admitted to the Affiliated Hospital of Qingdao University, Binzhou Medical University Hospital, Linyi People's Hospital, Shandong Provincial Hospital, Liaocheng People's Hospital, and the Maternal and Child Health Care of Zaozhuang between January 2012 and November 2015. The diagnosis of PE was based on the new-onset hypertension (systolic pressure ${ }^{3} 140 / 90 \mathrm{mmHg}$ or systolic pressure $\geq 90 \mathrm{mmHg}$ ) after 20 weeks of gestation, and accompanied by one of symptoms as follows: proteinuria $\left({ }^{3} 0.3 \mathrm{~g} / 24 \mathrm{~h}\right)$, urine protein/creatinine ratio ${ }^{3} 0.3$, random urine $\operatorname{protein}^{3}(+)$ or when not accompanied by symptoms of proteinuria occurring upper abdominal discomfort, headache, and blurred vision, according to previously published criteria[17]. We excluded patients with a history of hypertension, diabetes mellitus, or systemic lupus erythematosus. Inclusion criteria for control were as follows: 1) no clinical history of PE, chronic hypertension, heart disease, kidney disease, diabetes mellitus, hepatic diseases, transfusion, or immunotherapy; 2) without obstetric complications such as premature membrane rupture, placenta previa, threatened abortion, artificial insemination, twin or multiple pregnancies, and macrosomia in the present gestation. This study was approved by the Ethics Committee of the Affiliated Hospital of Qingdao University and obtained the written informed consent from all participants.

Taqman real-time fluorescence quantitative PCR 
Total DNA was extracted from peripheral venous blood and NOS2 rs2297518 genetic distribution was genotyped by the TaqMan real-time fluorescence quantitative polymerase chain reaction(PCR). The amplification method was performed according to previously published criteria[18]. The rs2297518 primers

were 5'- GTTGAGCTCTTTCAGCATGAAGAGC -3' (forward) and 5'-ATTTCTTCAGTTTCTAGAAAGAGAG -3'(reverse). Through fluorescence signal VIC and FAM to read results and then sanger sequencing to verify base represented by fluorescent signal.

\section{Statistical analysis}

SPSS 23.0 was used to data analysis. Student's t-test was used to compare differences in demographic and clinical characteristics between cases and controls. The chi-square test examined the Hardy-Weinberg equilibrium (HWE) in the control group to ensure that it was representative. Differences in genotypic and allelic frequencies between cases and controls were compared by Pearson's chi-square test.

\section{Results}

\section{Demographic and Clinical Characteristics}

Table 1 showed a comparison of demographic and clinical characteristics between PE patients and control groups. There were no significant differences in maternal age and age of menarche between PE patients and control groups ( $p>0.05)$. However, compared to the controls, gestational age admission(weeks), gravidity $(p=0.004)$, number of abortions $(p=0.006)$, birth weight, blood pressure, diastolic blood pressure, white blood cell and neutrophils ( $p=0.003)$ of the $P E$ group have significantly different $(p<0.001)$.

\section{Analysis of Genotypic and Allelic Frequencies}

The control group in our study satisfied the HWE (rs2297518, $\left.c^{2}=0.45, p=0.50\right)$. Table 2 showed the genetic distributions of NOS2 rs2297518 between PE and control groups. No significant differences were observed at this polymorphic site between the two groups in terms of genotypic distributions $\left(\chi^{2}=1.43, p=0.49\right)$, nor for allelic frequencies $\left(\chi^{2}=0.85, p=0.36, O R=1.07,95 \% \mathrm{Cl} 0.92-1.25\right)$.

To further investigate the relevance between NOS2 variant and PE, we divided PE group into severe PE group and non-severe PE group according to the diagnostic criteria of severe PE (pregnant women with PE have one of the following manifestations: systolic blood pressure $\geq 160 \mathrm{mmHg}$ and/or diastolic blood pressure $\geq 110 \mathrm{mmHg}$; increased level of AST or ALT; urine protein quantification $>2.0 \mathrm{~g} / 24 \mathrm{~h}$ and so on)[17]. The samples with incomplete clinical data were not grouped and the number is 23. Table 2 showed that no significant differences between severe/non-severe PE group and controls' genetic distributions were found at this polymorphic site (non-severe PE vs. control: $\chi^{2}=1.94, p=0.89$ by genotype, $\chi^{2}=1.49, p=0.65$ by allele; severe PE vs. control: $\chi^{2}=0.23, p=0.33$ by genotype, $\chi^{2}=0.21, p=0.22$ by allele).

Early-onset PE patients were those diagnosed before the 34th week of gestation, and are known to be more severely affected than those with later-onset PE [19]. Similarly, there are 5 samples of incomplete clinical data were not grouped. Table 2 showed that there were also no significant differences in the genetic distributions of rs2297518 between early/late-onset $P E$ and control groups (early-onset PE vs. control: $\chi^{2}=1.90, p=0.39$ by genotype, $\chi^{2}=0.012$, $p=0.91$ by allele; late-onset $P E$ vs. control: $\chi^{2}=1.82, p=0.40$ by genotype, $\chi^{2}=1.82, p=0.18$ by allele).

\section{Discussion}

Preeclampsia is a serious pregnancy complication threatening the health of mother and fetus and can even cause death. Insufficient trophoblast cell invasion induces incomplete spiral artery remodeling and further leads to hysteroplacental hypoperfusion, which are main pathological manifestations of PE. The pathogenesis of PE may involve multiple pathological changes such as excessive systemic inflammation, oxidative stress, vasospasm, change of cytokines, but is currently unclear.

As an effective vasodilator, NO is involved in the blood pressure regulation of PE, and is activated by three NOSs including neuronal NOS (nNOS), endothelial NOS (eNOS) and inducible NOS (iNOS). iNOS and eNOS are found in placenta. Unlike the other two NOSs, iNOS can function independently of calcium or calmodulin[20]. The iNOS was found to express on villous mesenchymal cells by iNOS-specific monoclonal antibody[21]. iNOS expression also can be detected in the vascular endothelial cells under the stimulation of inflammation[22]. Uncomplicated pregnancy is itself in the state of oxidative stress[23], active species superoxide will react with NO to produce peroxynitrite in the development of PE. iNOS can produce NO, thus involve the process of oxidative stress, which may be related to the occurrence of PE. Moreover, pregnancy itself will increase the expression of iNOS in smooth muscle cells, suggesting iNOS may be related to the regulation of uterine muscle tone [24]. iNOS expression is related to the release of free radicals and cytokines involved in PE [25, 26]. Under the condition of inflammatory, iNOS may active more NO and be involve in the pathological process of PE. Besides, iNOS is concerned with the regulation of adaptive changes in renal hemodynamics during pregnancy, which may induce the increased blood pressure in PE[2]. Du [27]et.al suggested enhancive iNOS may facilitate endoplasmic reticulum stress that promoted apoptosis of placenta trophoblast cells in PE. Mazzant et.al [28] reported that the level of iNOS presented an evident increase in 30 women in a PE group compared with 30 healthy pregnant women. Besides, Mao et.al[29] found that apoErat and apoE-/iNOS- rat have an obvious increase in blood pressure compared with wild type (WT). What's more, the increase in blood pressure in the apoE-/iNOS- rat is higher than the apoE- rat, indicating iNOS may be involved in the pathogenesis of PE related to dyslipidemia.

The onset of PE is regulated by multiple genes, and the SNPs (single nucleotide polymorphisms)s of candidate genes were closely related to susceptibility of PE. Some SNPs of candidate genes related to oxidative stress such as XRCC1, CypA, SOD2 and others showed a statistically significant association with 
PE[30-32], among them, the polymorphisms of NOS2 play an important role in the development of PE[33]. BHATNAGAR et.al found G300A located in exon 8 and G274T in exon 16 of NOS2 were associated with the occurrence of PE[33] in the population of North India. However, NOS2 rs2779249(-1026C/A) could enhance transcriptional activity of the iNOS promoter and further promote the production of NO, although no obvious difference was found in this polymorphism between PE patients and healthy pregnancy women[34]. Therefore, we analyzed the rs2297518 polymorphism, located in the exon 6 of NOS2 in chromosome 17, that causes an amino acid substitution from serine to leucine and an increase in NOS2 activity, and even alters the NOS2 protein function[35]. Previous studies have shown this polymorphism could affect the susceptibility to many disease conditions, such as inflammatory bowel disease, migraine with aura and so on[35]. Bhatnagar et al. analyzed the polymorphisms of the NOS2 were associated with hypertensive disorders of pregnancy(HDP), especially with PE[35]. Amaral[8] analyzed the genotypes and alleles frequency of NOS2 rs2297518 in 212 healthy pregnant women and 187 PE patients of Brazil population, and found that the GA allele frequency and A allele frequency expresses an obvious increase in PE group. However, a Finland cohort study found no obvious relationship between rs2297518 and the occurrence of hypertension[14]. In our study, we explored the relevance between PE susceptibility and NOS2 rs2297518 from 979 PE patients and 1187 normal pregnant women in Chinese Han population, indicating that there were no significant differences in genotypic and allelic frequencies of this SNP between PE and control groups. To further understand the relationship between NOS2 and PE, we divided the $\mathrm{PE}$ patients into mild/severe and early/late-onset groups, the results showed that there were also no significant differences in genetic distributions at the polymorphic sites between mild/severe PE and control groups, or between early/late-onset PE and control groups. These data suggested that the rs2297518 in NOS2 may not play a pivotal role in the pathophysiology of PE in Chinese Han women. This study was the first to explore the relevance between NOS2 rs2297518 and susceptibility of PE in the Chinese Han women.

In conclusion, our results showed that the rs2297518 polymorphism in NOS2 was not associated with PE in Chinese Han women. Even though PE is a complex polygenetic hereditary disease and our research involves large-scale clinical data, the results may be influenced by the multiple races and regions, which need to expand the sample size in a future study. In addition, we only explored the rs 2297518 polymorphism in NOS 2 but did not analyze the interaction with other polymorphisms. Therefore, genetic studies in other candidate genes for PE from different races and regions are required to detect the possibly different pathophysiological mechanisms, which will increase our understanding of the pathogenesis of PE and further to explore methods of targeted therapy relating to gene changes between populations.

\section{Declarations}

Funding: This work was supported by the National Natural Science Foundation of China [No. 81600211]; Natural Science Fund Project of Shandong Province [ZR2019MH127]; Key research and development plan of Shandong Province [2019GSF108106] and the Application and Basic Research Project of Qingdao[182-2-27-jch].

Conflicts of interest: The authors declare that they have no conflict of interest.

Code availability: All softwares involved in this study are listed in article.

Authors' contributions: Jingli Wang and Chengcheng Guan wrote the main body of this manuscript. Huabing Hou and Xinguo Peng collected much literature about this manuscript. Yun Liu revised and corrected this manuscript. Jinling Li designed this study.

Ethics approval: This study was approved by the Ethics Committee of the Affiliated Hospital of Qingdao University.

Consent to participate: The written informed consents were obtained from all participants.

Consent for publication: All authors agree to publish.

\section{References}

1. Duley L (2009) The global impact of pre-eclampsia and eclampsia. Semin Perinatol 33(3):130-137. doi:10.1053/j.semperi.2009.02.010 Epub $2009 / 05 / 26$.

2. S0146-0005(09)00021 - 4 [pii]. PubMed PMID: 19464502

3. Alexander B, Cockrell K, Cline F, Granger J. Inducible nitric oxide synthase inhibition attenuates renal hemodynamics during pregnancy. Hypertension (Dallas, Tex: 1979). 2002;39:586 - 90. doi: 10.1161/hy0202.103288. PubMed PMID: 11882613

4. Jeyabalan A, Conrad K (2007) Renal function during normal pregnancy and preeclampsia. Frontiers in bioscience: a journal virtual library 12:2425-2437 doi: 10.2741/2244. PubMed PMID: 17127252

5. Alexander B, Miller M, Kassab S, Novak J, Reckelhoff J, Kruckeberg W et al Differential expression of renal nitric oxide synthase isoforms during pregnancy in rats. Hypertension (Dallas, Tex: 1979) 1999;33:435-9. doi: 10.1161/01.hyp.33.1.435. PubMed PMID: 9931143

6. Pautz A, Art J, Hahn S, Nowag S, Voss C, Kleinert H (2010) Regulation of the expression of inducible nitric oxide synthase. Nitric oxide: biology and chemistry /. official journal of the Nitric Oxide Society 23(2):75-93 doi: 10.1016/j.niox.2010.04.007. PubMed PMID: 20438856

7. AJ T, JF T, IT GKAY, IA C (1997) G, et al. Nitric oxide synthase activity and localization do not change in uterus and placenta during human parturition. Human reproduction (Oxford England) 12(11):2546-2552. doi:10.1093/humrep/12.11.2546. PubMed PMID: 9436704

8. LM A, LC P, DA G, AC P, JT S, RL P et al (2013) Antihypertensive effects of inducible nitric oxide synthase inhibition in experimental pre-eclampsia. J Cell Mol Med 17(10):1300-1307. doi:10.1111/jcmm.12106. PubMed PMID: 23890248

9. Amaral LM, Palei AC, Sandrim VC, Luizon MR, Cavalli RC, Duarte G et al (2012) Maternal iNOS genetic polymorphisms and hypertensive disorders of pregnancy. J Hum Hypertens 26(9):547-552. doi:10.1038/jhh.2011.65 Epub 2011/07/01.

Page $4 / 6$ 
10. jhh201165 [pii]. PubMed PMID: 21716319

11. Sandrim V, Palei A, Cavalli R, Araújo F, Ramos E, Duarte G et al (2008) eNOS haplotypes associated with gestational hypertension or preeclampsia. Pharmacogenomics 9(10):1467-1473. doi:10.2217/14622416.9.10.1467. PubMed PMID: 18855535

12. Sandrim V, Palei A, Metzger I, Gomes V, Cavalli R, Tanus-Santos J (2008) Nitric oxide formation is inversely related to serum levels of antiangiogenic factors soluble fms-like tyrosine kinase-1 and soluble endogline in preeclampsia. Hypertension 52(2):402-407.

doi:10.1161/hypertensionaha.108.115006. PubMed PMID: 18574068

13. Hubel C. Oxidative stress in the pathogenesis of preeclampsia. Proceedings of the Society for Experimental Biology and Medicine Society for Experimental Biology and Medicine (New York, NY). 1999;222(3):222 - 35. doi: 10.1046/j.1525-1373.1999.d01-139.x. PubMed PMID: 10601881

14. W LDFHLK (2017) T, Y L, D C. eNOS/iNOS and endoplasmic reticulum stress-induced apoptosis in the placentas of patients with preeclampsia. J Hum Hypertens 31(1):49-55. doi:10.1038/jhh.2016.17. PubMed PMID: 27030287

15. Faxén M, Nisell H, Kublickiene K (2001) Altered mRNA expression of ecNOS and iNOS in myometrium and placenta from women with preeclampsia. Archives of gynecology obstetrics 265(1):45-50 doi: 10.1007/s004040000152. PubMed PMID: 11327094

16. Nikkari S, Määttä K, Kunnas T. Functional Inducible Nitric Oxide Synthase Gene Variants Associate With Hypertension: A Case-Control Study in a Finnish Population-The TAMRISK Study. Medicine. 2015;94(46):e1958. doi: 10.1097/md.0000000000001958. PubMed PMID: 26579803

17. Thomson A, Telfer J, Kohnen G, Young A, Cameron I, Greer I et al (1997) Nitric oxide synthase activity and localization do not change in uterus and placenta during human parturition. Human reproduction (Oxford England) 12(11):2546-2552. doi:10.1093/humrep/12.11.2546. PubMed PMID: 9436704

18. Amaral L, Pinheiro L, Guimaraes D, Palei A, Sertório J, Portella R et al (2013) Antihypertensive effects of inducible nitric oxide synthase inhibition in experimental pre-eclampsia. J Cell Mol Med 17(10):1300-1307. doi:10.1111/jcmm.12106. PubMed PMID: 23890248

19. ACOG Practice Bulletin No. 202: Gestational Hypertension and Preeclampsia. Obstetrics and gynecology. 2019;133(1):1. doi: 10.1097/aog.0000000000003018. PubMed PMID: 30575675

20. Wang H, Guo M, Liu F, Wang J, Zhou Z, Ji J et al (2015) Role of IL-17 Variants in Preeclampsia in Chinese Han Women. PloS one 10(10):e0140118. doi:10.1371/journal.pone.0140118. PubMed PMID: 26451724

21. Alvarez-Fernandez I, Prieto B, Rodriguez V, Ruano Y, Escudero Al, Alvarez FV (2014) New biomarkers in diagnosis of early onset preeclampsia and imminent delivery prognosis. Clinical chemistry laboratory medicine: CCLM / FESCC 52(8):1159-1168. doi:10.1515/cclm-2013-0901. PubMed PMID: 24516004

22. Stuehr D, Kwon N, Gross S, Thiel B, Levi R, Nathan C (1989) Synthesis of nitrogen oxides from L-arginine by macrophage cytosol: requirement for inducible and constitutive components. Biochem Biophys Res Commun 161(2):420-426. doi:10.1016/0006-291x(89)92615-6. PubMed PMID: 2735902

23. Myatt L, Eis A, Brockman D, Kossenjans W, Greer I, Lyall F (1997) Inducible (type II) nitric oxide synthase in human placental villous tissue of normotensive, pre-eclamptic and intrauterine growth-restricted pregnancies. Placenta 18(4):261-268. doi:10.1016/s0143-4004(97)80060-4. PubMed PMID: 9179919

24. Palmer R. The discovery of nitric oxide in the vessel wall. A unifying concept in the pathogenesis of sepsis. Archives of surgery (Chicago, III: 1960). 1993;128(4):396-401. doi: 10.1001/archsurg.1993.01420160034004. PubMed PMID: 7681276

25. Myatt L, Cui X. Oxidative stress in the placenta. Histochemistry and cell biology. 2004;122(4):369 - 82. doi: 10.1007/s00418-004-0677-x. PubMed PMID: 15248072

26. Bansal R, Goldsmith P, He Y, Zaloudek C, Ecker J, Riemer R (1997) A decline in myometrial nitric oxide synthase expression is associated with labor and delivery. J Clin Investig 99(10):2502-2508. doi:10.1172/jci119434. PubMed PMID: 9153294

27. Hecker M, Cattaruzza M, Wagner A (1999) Regulation of inducible nitric oxide synthase gene expression in vascular smooth muscle cells. Gen Pharmacol 32(1):9-16. doi:10.1016/s0306-3623(98)00082-2. PubMed PMID: 9888247

28. Chu S, Marks-Konczalik J, Wu H, Banks T, Moss J (1998) Analysis of the cytokine-stimulated human inducible nitric oxide synthase (iNOS) gene: characterization of differences between human and mouse iNOS promoters. Biochem Biophys Res Commun 248(3):871-878. doi:10.1006/bbrc.1998.9062. PubMed PMID: 9704020

29. Du L, He F, Kuang L, Tang W, Li Y, Chen D (2017) eNOS/iNOS and endoplasmic reticulum stress-induced apoptosis in the placentas of patients with preeclampsia. J Hum Hypertens 31(1):49-55. doi:10.1038/jhh.2016.17. PubMed PMID: 27030287

30. Mazzanti L, Raffaelli F, Vignini A, Nanetti L, Vitali P, Boscarato V et al (2012) Nitric oxide and peroxynitrite platelet levels in gestational hypertension and preeclampsia. Platelets 23(1):26-35. doi:10.3109/09537104.2011.589543. PubMed PMID: 21787174. Epub 2011/07/27.

31. Mao L, Zhou Q, Zhou S, Wilbur R, Li X (2013) Roles of apolipoprotein E (ApoE) and inducible nitric oxide synthase (iNOS) in inflammation and apoptosis in preeclampsia pathogenesis and progression. PloS one 8(3):e58168. doi:10.1371/journal.pone.0058168. PubMed PMID: 23472151

32. Sun W, Xu Y, Xin Q, Zhang Y, Cui B, Hong F (2019) Association between polymorphism in Cyclophilin A gene and its serum and placental expression in Han Chinese women with severe preeclampsia. Pregnancy hypertension 15:84-92 doi: 10.1016/j.preghy.2018.11.005. PubMed PMID: 30825933

33. Luo Z, Julien P, Wei S, Audibert F, Fraser W (2018) Association of pre-eclampsia with SOD2 Ala16Val polymorphism among mother-father-infant triads. Int J Gynaecol Obstet 142(2):221-227. doi:10.1002/ijgo.12528. PubMed PMID: 29745991

34. Saadat I, Beyzaei Z, Aghaei F, Kamrani S, Saadat M (2012) Association between polymorphisms in DNA repair genes (XRCC1 and XRCC7) and risk of preeclampsia. Archives of gynecology obstetrics 286(6):1459-1462. doi:10.1007/s00404-012-2471-7. PubMed PMID: 22825692

35. Bhatnagar S, Bhattacharjee J, Vaid M, Madan T, Trivedi S, Sarma P. Inducible nitric oxide synthase (iNOS) gene polymorphism in pre-eclampsia: a pilot study in North India. The Australian \& New Zealand journal of obstetrics \& gynaecology. 2007;47(6):477 - 82. doi: 10.1111/j.1479-828X.2007.00783.X. PubMed PMID: 17991113 
36. Amaral L, Palei A, Sandrim V, Luizon M, Cavalli R, Duarte G et al (2012) Maternal iNOS genetic polymorphisms and hypertensive disorders of pregnancy. J Hum Hypertens 26(9):547-552. doi:10.1038/jhh.2011.65. PubMed PMID: 21716319

37. Dhillon S, Mastropaolo L, Murchie R, Griffiths C, Thöni C, Elkadri A et al (2014) Higher activity of the inducible nitric oxide synthase contributes to very early onset inflammatory bowel disease. Clinical translational gastroenterology 5:e46. doi:10.1038/ctg.2013.17. PubMed PMID: 24430113

\section{Tables}

Table 1.Demographic and clinical characteristics of the two groups( $x \pm s)$

\begin{tabular}{|c|c|c|c|c|c|c|c|c|c|c|c|c|c|c|c|}
\hline \multicolumn{6}{|c|}{ Characteristics } & \multicolumn{2}{|c|}{ PE(979ه } & \multicolumn{2}{|c|}{ Control(1187区 } & \multicolumn{2}{|l|}{$\mathrm{t}$} & p-value & \multicolumn{3}{|c|}{ Note:The clinical characteristic of } \\
\hline \multicolumn{6}{|c|}{ Maternal age(years) } & \multicolumn{2}{|c|}{$30.12 \pm 5.76$} & \multicolumn{2}{|c|}{$30.31 \pm 4.09$} & \multicolumn{2}{|c|}{0.876} & 0.381 & $\begin{array}{l}\text { ivery } N \\
\text { lyzed b }\end{array}$ & $\begin{array}{l}\text { ethod" v } \\
c^{2} \text { test }\end{array}$ & $\begin{array}{l}\text { atistical } \\
\text { thers by }\end{array}$ \\
\hline \multicolumn{6}{|c|}{ Gestational age at admission } & \multicolumn{2}{|c|}{$35.16 \pm 3.64$} & \multicolumn{2}{|c|}{$39.03 \pm 1.62$} & \multicolumn{2}{|c|}{30.07} & $<0.0001$ & \multicolumn{3}{|c|}{ student's t test. } \\
\hline \multicolumn{6}{|c|}{ Gestational age at delivery(weeks) } & \multicolumn{2}{|c|}{$35.84 \pm 3.31$} & \multicolumn{2}{|c|}{$39.36 \pm 1.34$} & \multicolumn{2}{|c|}{29.99} & $<0.0001$ & & & \\
\hline \multicolumn{6}{|l|}{ Gravidity } & \multicolumn{2}{|c|}{$2.55 \pm 3.27$} & \multicolumn{2}{|c|}{$2.23 \pm 1.17$} & -2.9 & & 0.004 & & & \\
\hline Number of a & ortior & & & & & $0.66 \pm$ & .98 & & & -0.2 & & 0.006 & $\begin{array}{l}\text { le 2. Th } \\
\text { etic dis }\end{array}$ & $\begin{array}{l}\text { compa } \\
\text { ribution }\end{array}$ & $\begin{array}{l}\text { of } \\
\text { os2 }\end{array}$ \\
\hline Systolic bloc & pres & ure $(\mathrm{mmHc}$ & & & & 159.7 & \pm 18.65 & & +10.06 & -68 & & $<0.0001$ rs 2 & 97518 & polymor & \\
\hline Diastolic blo & d pre & sure $(\mathrm{mmH}$ & & & & 104.3 & \pm 13.55 & & 7.82 & -63 & & $<0.0001^{b}$ & veen $\mathrm{Pl}$ & Early-o & E, Late- \\
\hline Birth weights & & & & & & 2420. & $8 \pm 897$ & & $7 \pm 587.14$ & -21 & 25 & $<0.0001$ gro & & 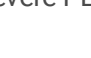 & \\
\hline White blood & ell $(W$ & C) $\left(\times 10^{9 / L}\right)$ & & & & $9.86 \pm$ & .71 & & & -5.8 & & $<0.001$ & & & \\
\hline Neutrophils( & $(\times 10$ & & & & & $7.43 \pm$ & 60 & & & -2.9 & & 0.003 & & & \\
\hline Age of mena & che & & & & & 14.02 & 1.18 & & 1.27 & 0.6 & & 0.062 & & & \\
\hline Triglycerides & $\mathrm{mmo}$ & & & & & $3.80 \pm$ & 1.70 & & & 16. & & $<0.0001$ & & & \\
\hline Total choles & $\mathrm{rol}(\mathrm{n}$ & $\mathrm{nol} / \mathrm{L})$ & & & & $6.80 t$ & 2.40 & & & 4.1 & & $<0.0001$ & & & \\
\hline Alanine tran & $\min$ & $\mathrm{e}(\mathrm{ALT})(\mathrm{IU}$ & & & & $23.4 C$ & $\$ 34.50$ & & & 8.2 & & $<0.0001$ & & & \\
\hline Aspartate an & notra & sferase (A & T) $(I U / L$ & & & 33.60 & 145.60 & & & 3.1 & & 0.002 & & & \\
\hline Creatinine $(u$ & $\mathrm{hol} / \mathrm{L}$ & & & & & 60.00 & 26.00 & & & 7.9 & & $<0.0001$ & & & \\
\hline Delivery Met & ods & & & & & & & & & & & & & & \\
\hline Normal deliv & & & & & & $96(10$ & $6 \%)$ & & $4 \%)$ & & & $<0.0001$ & & & \\
\hline Cesarean se & ion & & & & & $809(8$ & $.4 \%)$ & & $.4 \%)$ & & & & & & \\
\hline & & No. of & PE cas & (979) & & $\begin{array}{l}\text { Non- } \\
(171)\end{array}$ & evere PE & & Severe $\mathrm{P}$ & DE cas & (808) & $\begin{array}{l}\text { Early } \\
(487)\end{array}$ & onset $P$ & case & $\begin{array}{l}\text { Late-on: } \\
(510)\end{array}$ \\
\hline & & ه1187ه & No & $P^{1}$ & OR & No & $P$ & & No & $P$ & OR & No & $P$ & & No \\
\hline & & & & & $\begin{array}{l}(95 \% \\
\mathrm{Cl})\end{array}$ & & & & & & $\begin{array}{l}(95 \% \\
\mathrm{Cl})\end{array}$ & & & $\begin{array}{l}(95 \% \\
\mathrm{Cl})\end{array}$ & \\
\hline Genotype & AA & 26 & 15 & 0.49 & & 4 & 0.89 & & 11 & 0.33 & & 6 & 0.39 & & 9 \\
\hline & $A G$ & 280 & 225 & & & 43 & & & 182 & & & 120 & & & 105 \\
\hline & GG & 881 & 739 & & & 124 & & & 615 & & & 351 & & & 388 \\
\hline Total & & 1187 & 979 & & & 171 & & & 808 & & & 477 & & & 502 \\
\hline $\begin{array}{l}\text { Allele } \\
\text { frequencies }\end{array}$ & A & 332 & 255 & 0.36 & 1.08 & 51 & 0.65 & & 204 & 0.22 & 1.10 & 132 & 0.91 & 1.01 & 123 \\
\hline & & & & & $\begin{array}{l}(0.92- \\
1.25)\end{array}$ & & & & & & $\begin{array}{l}(0.94- \\
1.30)\end{array}$ & & & $\begin{array}{l}(0.84- \\
1.22)\end{array}$ & \\
\hline & $G$ & 2042 & 1703 & & & 291 & & & 1412 & & & 822 & & & 881 \\
\hline & & & & & & & & & & & & & & & \\
\hline Total & & 2374 & 1958 & & & 342 & & & 1616 & & & 954 & & & 1004 \\
\hline
\end{tabular}

${ }^{1}$ The chi-square test were used to estimate p-values, odds ratios and $95 \%$ confidence intervals. 